\title{
Deciphering the molecular basis of invasiveness in Sdhb- deficient cells
}

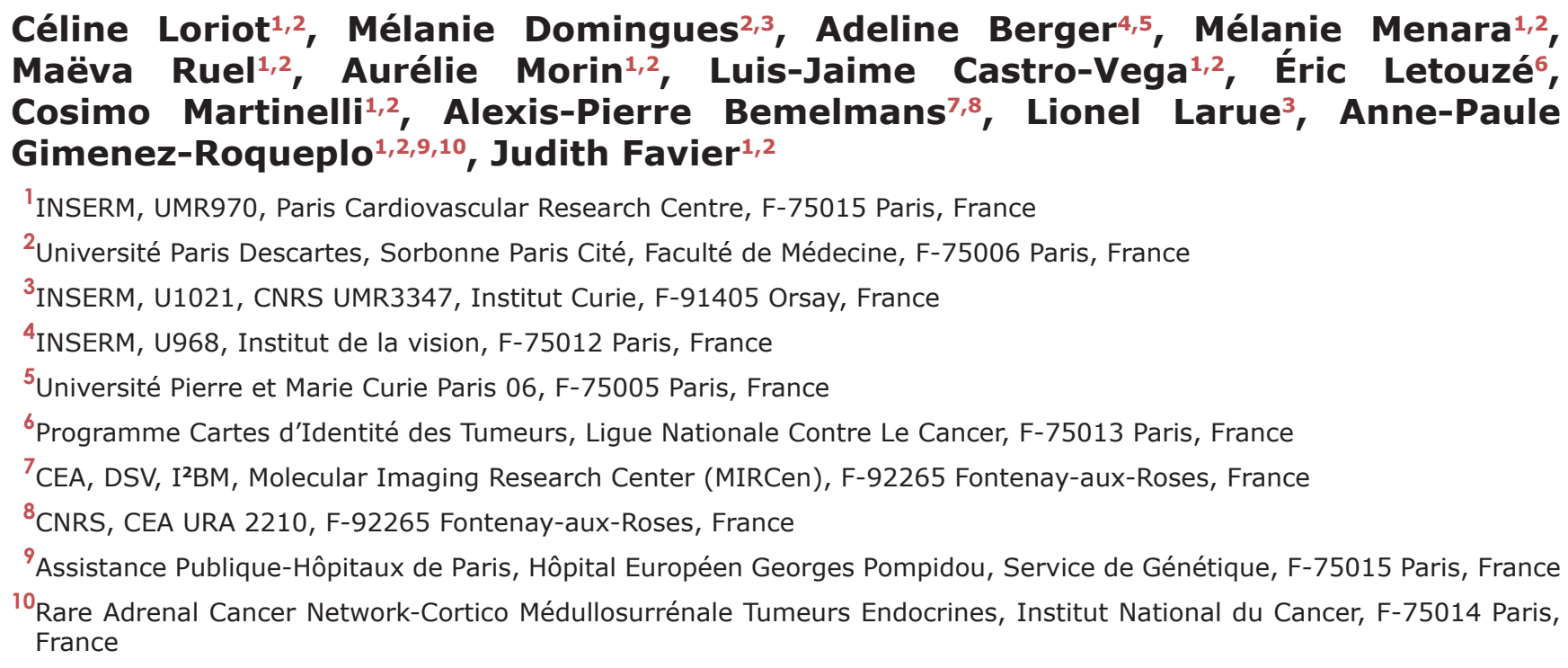

Correspondence to:

Judith Favier, e-mail: judith.favier@inserm.fr

Keywords: paraganglioma, SDHB, metastasis, EMT, keratin 19

Received: May 11,2015 Accepted: September 25, $2015 \quad$ Published: October 08, 2015

\section{ABSTRACT}

Metastatic pheochromocytomas and paragangliomas (PPGL) are malignant neuroendocrine tumors frequently associated with germline mutations in the SDHB gene. SDHB-mutated PPGL display a hypermethylator phenotype associated with hallmarks of epithelial-to-mesenchymal transition (EMT). In the present study, we report the characterization of a unique model of Sdhb knockout in mouse chromaffin cells. Sdhb deficient cells exhibit a metastatic phenotype as highlighted by increased individual cell migration (characterized by faster motility and increased persistence) as well as high invasive and adhesion abilities. This phenotype is associated with the modulation of Twist1, Twist2, Tcf3, Snai1, N-cadherin or Krt19 expression, reflecting an EMT-like reprogramming of cells. Krt19 is epigenetically silenced in Sdhb-deficient cells and re-expressed after treatment by the demethylating agent decitabine. Krt19 rescue by lentiviral transduction in Sdhb-deficient cells and Krt19 inhibition by RNA interference in wild-type cells were performed. Both studies revealed the involvement of KRT19 in the invasive phenotype by modulating collective and individual migration and cell/extra-cellular matrix adhesion properties. These findings underline the role of hypermethylation and EMT in the in vitro acquisition of metastatic properties, following SDHB loss of function.

\section{INTRODUCTION}

Pheochromocytomas and paragangliomas (PPGL) are rare neuroendocrine tumors that arise from chromaffin cells of the adrenal medulla and from sympathetic or parasympathetic ganglia, respectively. In about $40 \%$ of cases, PPGL are inherited, which implies that a germline mutation has been identified in one of the 13 known PPGL predisposition genes: the RET and $H I F 2 A$ proto-oncogenes and the NF1, VHL, SDHx (SDHA, SDHB, SDHC, SDHD and $S D H A F 2$ ), MAX, TMEM127, FH and MDH2 tumor suppressor genes [1,2]. Somatic mutations in NF1, VHL, 
MAX, HIF $2 A$ and HRAS genes have also been identified in around $30 \%$ of cases [3].

Among PPGL susceptibility genes, $S D H B$ is specifically associated with malignancy and poor prognosis. Patients carrying an $S D H B$ mutation are more likely to develop a metastatic form of their disease with a median survival substantially reduced, compared to patients without $S D H B$ mutation [4]. Additionally, time lapse between diagnosis and development of metastasis is shorter for $S D H B$ mutation carriers than others (for whom it can last up to 20 years). However, it remains unclear why $S D H B$-mutation carriers are predisposed to these aggressive forms of the disease and genomic studies have failed in identifying a causative genomic event, concomitant to SDHB loss, that would explain this peculiar phenotype $[5,6]$.

We previously proposed epithelial-to-mesenchymal transition (EMT) as the process likely to be responsible for $S D H B$-related malignancy in PPGL. Using transcriptome profiling of a large cohort of PPGL, we reported that $S D H B$-malignant samples displayed an overexpression of LOXL2, TWIST1, TCF3, MMP1, MMP2 and a down regulation of $C D H 2$ and $K R T 19$, which are all hallmarks of the EMT process. Furthermore, the nuclear retention of SNAIL was specifically observed in $S D H B$ malignant tumors, suggesting an active form of this key EMT transcription factor in this subgroup of aggressive tumors [7].

EMT is a physiological embryonic process that allows, among others, neural crest cells migration after neural tube closure. It can also be found in pathological conditions such as fibrosis [8] and metastatic dissemination [9]. Cancer cells, which are submitted to EMT undergo a myriad of morpho-physiological and epigenetic changes, including but not limited to the loss of cell junctions, increased proteolytic abilities and enhanced migratory and invasive capacities [10]. During malignant progression, tumor cells lose cell/cell and cell/extracellular matrix adhesion properties, which can be assessed by decreased actin-dependent focal adhesion or keratins-dependent hemi desmosomes [11]. Keratins (KRT, also called cytokeratins) are part of intermediate filaments. Among the 54 keratins [12], keratin 19 (KRT19) is the smallest one, and is of peculiar interest because of its implication as a diagnostic biomarker of circulating cells in breast cancer [13]. Loss or down regulation of KRT19 is associated with an aggressive behavior characterized by enhanced migratory, adhesive and invasive properties in squamous cell carcinomas, neuroblastomas, renal and breast cancers [14-19]. KRT19 extinction is due to the methylation of its promoter, as described in uterine leiomyoma, renal cell carcinoma, as well as in neuroblastoma, another neural crest cell derived tumor $[17,20,21]$. This was described to be associated with high-grade tumors, and thus with reduced patients' survival $[15,16]$.
We and others, recently demonstrated that mutations in $S D H x$ genes (encoding succinate dehydrogenase subunits) lead to succinate accumulation, which inhibits DNA demethylases (TET enzymes), leading to a global hypermethylation of DNA [22-25]. In SDHB-mutated PPGL and in $S d h b^{-/} /$immortalized mouse chromaffin cells (imCC), we demonstrated that KRT19 was one of the most hypermethylated and down-regulated gene [24].

Following these observations, we evaluated here the consequences of SDHB loss and the role of KRT19 in the establishment of a metastatic phenotype following in vitro inactivation of $S d h b$ in murine chromaffin cells.

\section{RESULTS}

\section{Sdhb-/- immortalized mouse chromaffin cells display an EMT-like metastatic phenotype}

We have previously reported the initial characterization of the $S d h b^{-} /-$imCC cell line, which displays a hypermethylator phenotype associated with increased collective migration capacities and a reduced proliferation [24]. Observation of cell morphology revealed a mesenchymal aspect of $S d h b-/-$ cells, reminiscent of an EMT phenotype (Supplementary Figure 1). Individual cell migration assessed by single cell tracking analysis revealed that $S d h b^{-} /-$imCC migrate at a mean speed of $22 \mu \mathrm{m} / \mathrm{h}$, which is significantly faster than control cells $(17 \mu \mathrm{m} / \mathrm{h})$ (Figure 1A and Supplementary Movies 1 and 2). Consistently, as a result of enhanced individual cell migration, total distance was significantly higher in $S d h b-/-$ imCC compared to wild-type (WT) cells. Interestingly, vector displacement diagrams revealed that in contrast with WT cells which tend to go round in circles, $S d h b$-deficient cells aim to move away from their point of origin and follow a linear path (Figure 1B), further increasing the travelled distance or persistence (Figure 1C).

We next performed inverted invasion assays in a Matrigel ${ }^{\circledR}$ matrix, to determine whether $S d h b-/-$ imCC are able to actively pass through an $8 \mu \mathrm{m}$ filter, digest Matrige $\mathrm{l}^{\circledR}$ matrix, and migrate beyond a $30 \mu \mathrm{m}$ arbitrary limit, classically used for such experiments (Figure 1D). Very few WT cells were able to get through the filter, while $25 \%$ of $S d h b-/-$ imCC did (Figure 1E). Accordingly, about $60 \%$ of the latter were then able to migrate beyond the arbitrary $30 \mu \mathrm{m}$ limit; while none of the WT cells did (Figure 1F). Interestingly, $S d h b-/-$ imCC were found up to $180 \mu \mathrm{m}$ after the filter, with a $90 \mu \mathrm{m}$ mean distance, compared to $20 \mu \mathrm{m}$ for WT cells (Figure 1G), showing real skills to progress into the matrix.

The ability of passing through the filter, digesting extracellular matrix, coupled with high migration rates mimic the different steps that occur during the process of intravasation (or trans-endothelial migration). We then 
A
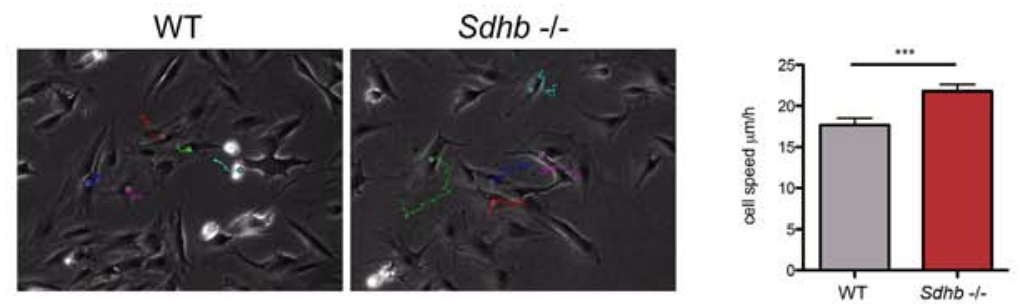

B

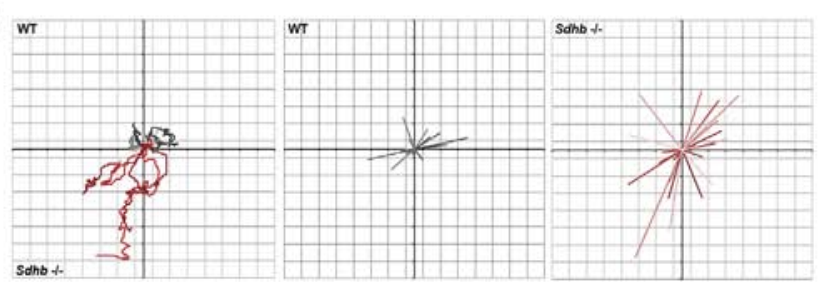

C

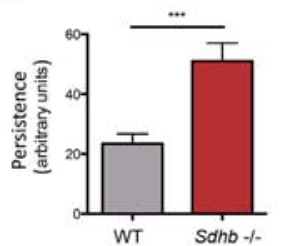

D
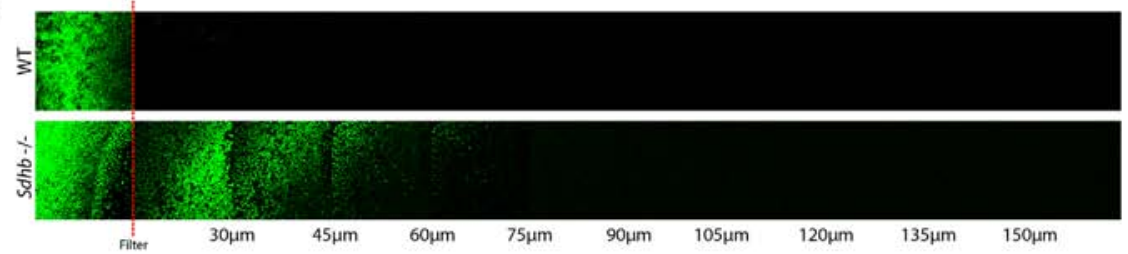

E

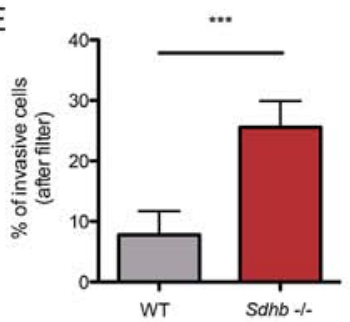

H
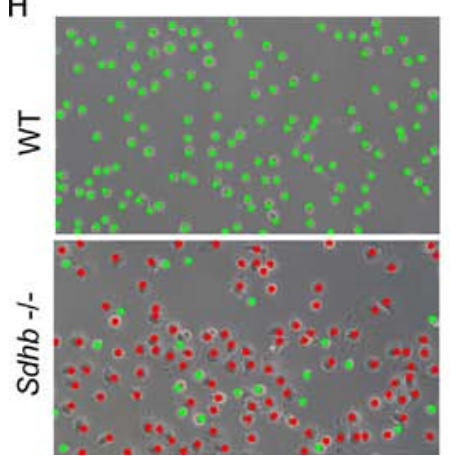

F

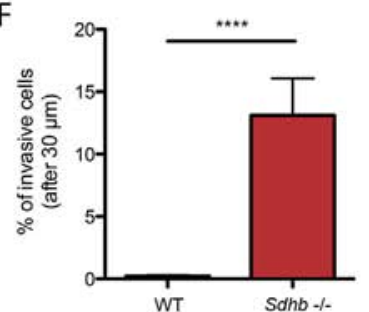

G

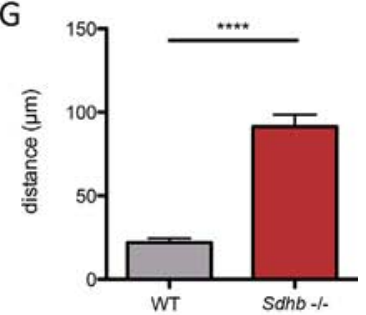

I

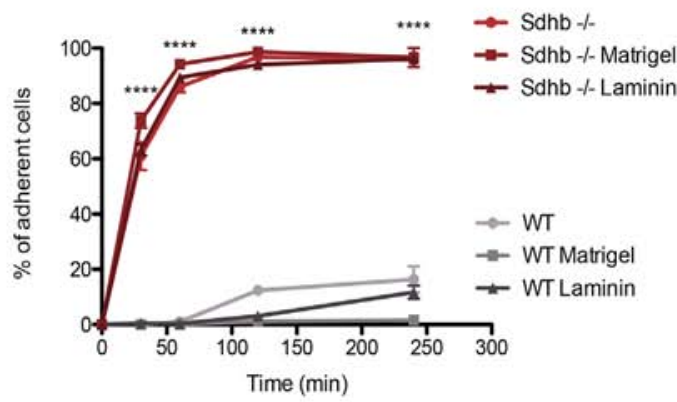

Figure 1: Characterization of changes in migratory and invasive properties following $\boldsymbol{S d h b}$ loss. A. Individual cells speed was estimated by tracking cell nucleus during 12 hours. ${ }^{* * *} p=0.0003$. B. Vector displacement diagrams representing total distance (left panel), and persistence (middle and right panel). C. Comparison of persistence. $* * * p=0.007$. D. Cells invasion's abilities were evaluated through their capacity to move into a Matrigel matrix. Red line symbolized the filter. E. Quantification of cell abilities to pass through the filter. ${ }^{* *} p=0.004$. F. Quantification of cell invasion, cells were considered invasive beyond $30 \mu \mathrm{m} . * * * * p<0.0001$. G. Mean distance travelled by cells into Matrigel matrix. ${ }^{* * * *} p<0.0001$. H. Cell adhesion abilities were measured by counting adherent (marked as red dots) versus non-adherent (marked as green dots) cells one hour after plating. Scale bar $125 \mu \mathrm{m} .{ }^{* * * *} p<0.0001$. I. Adhesion course: quantification of cell adhesion during 240 minutes. ${ }^{* * * *} p<0.0001$

See also Supplementary Movies 1 and 2.

looked at cell-substratum adhesion, without coating, or on plate coated with Matrigel ${ }^{\circledR}$ or laminin. We observed that cell adhesion is equivalent whatever the conditions used, and that one hour after plating, approximately
$90 \%$ of $S d h b^{-/}-$imCC are adherent whereas WT cells hardly adhere during the same period (Figures 1H and 1I). Three hours after plating, only about $20 \%$ of WT cells were adherent. 


\section{The EMT-like phenotype is associated with transcriptional reprogramming of $S d h b-/-$ immortalized mouse chromaffin cells}

Transcriptomic analysis of genes associated with EMT was performed to compare WT and $S d h b-/-$ imCC and revealed variations concordant with an activation of EMT in Sdhb-deficient cells (Figure 2A, and Supplementary Figure 2). Using qRT-PCR, we validated that the expression of the main markers of EMT, Twist1, Twist 2, Tcf 3 and Snail 1 were significantly increased, while $\mathrm{N}$-cadherin and Krt19's expression were significantly decreased in Sdhb-/- imCC (Figure 2B and 3C). The overexpression of TCF3 and SNAIL proteins was further demonstrated by western blot in Sdhb-deficient cells (Figure 2C) and immunofluorescence analysis showed the nuclear localization of SNAIL as well as down-regulation of N-cadherin and KRT19 proteins in $S d h b^{-/}-$imCC (Figure 2D and 3B).
Extravasation requires cells to roll and to adhere to endothelial cells, both process involving adhesion molecules such as integrins, galectins, focal adhesion kinases (FAK), selectins or mucins [26]. Interestingly, transcriptome analyses showed a significant overexpression of Cd24a, Mucl, Lgals3, Lgals3bp, Lgals9, Ptk2 and integrins $\alpha 1, \alpha 8, \alpha 11$, $\beta 3$ and $\beta 5$ mRNAs in $S d h b-/-$ imCC compared to WT cells (Supplementary Figure 3), underscoring the high adherent properties of $S d h b$-deficient cells. Moreover trans-endothelial migration, which requires the loss of adherent junctions between endothelial cells, or their retraction, can be promoted by $\beta$-catenin and VEGF [26]. Interestingly, we observed that both Ctnnb1 and Vegfa mRNA were overexpressed in $S d h b-/-$ imCC (Supplementary Figure 3). Altogether, these data established that loss of SDHB promotes a transcriptional reprogramming that is associated with an EMT phenotype in the mouse chromaffin cell line.
A

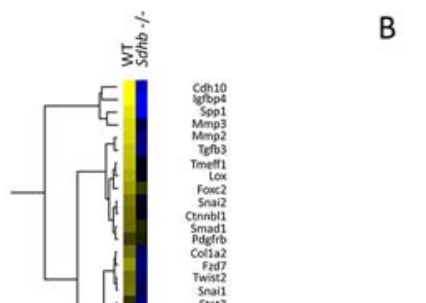

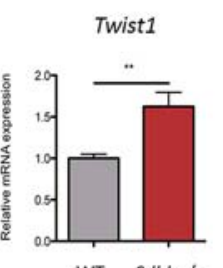

WT $S d h b \%$
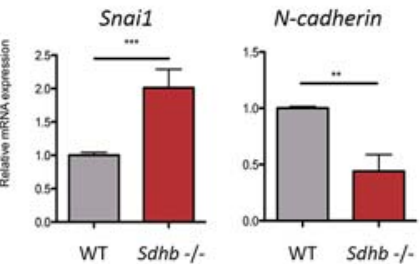

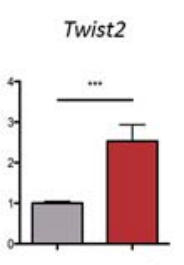

WT Sdhb -\%

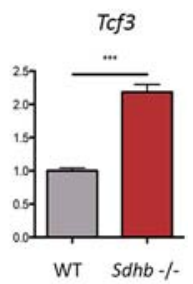

D

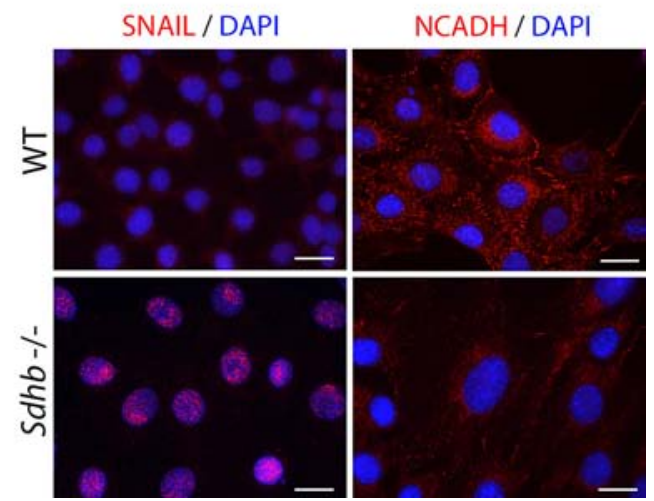

Figure 2: Molecular characterization of the EMT process in $\boldsymbol{S d h b - / - ~ i m C C . ~ A . ~ H e a t ~ m a p ~ s a m p l e s ~ o f ~ t h e ~ m o s t ~ d i f f e r e n t i a l l y ~}$ expressed EMT associated genes between WT and Sdhb-/- imCC. B. qRT-PCR analyses showing Twist1, Twist2, Tcf3, and Snail inductions as well as and $N$-cadherin loss of expression in $S d h b$ deficient imCC compared to WT cells. $* * p=0.0079$ and $p=0.0099$; ${ }^{* * *} p=0.0002$. Data are shown as fold change relative to WT C. SNAIL and TCF3 were studied at protein level using western blotting and specific antibodies. D. SNAIL and N-Cadherin were studied using immunofluorescence and specific antibodies. Scale bar $20 \mu \mathrm{m}$ See also Supplementary Figures 2 and 3 


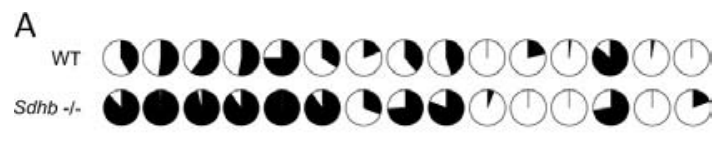

B
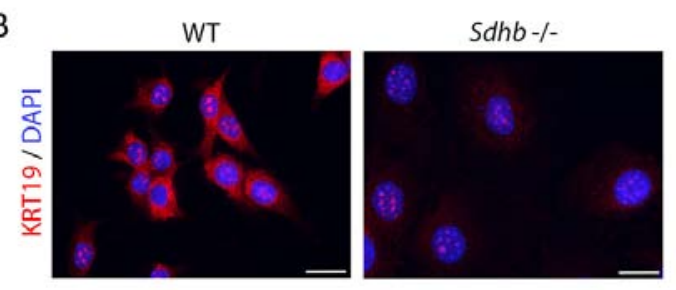

C

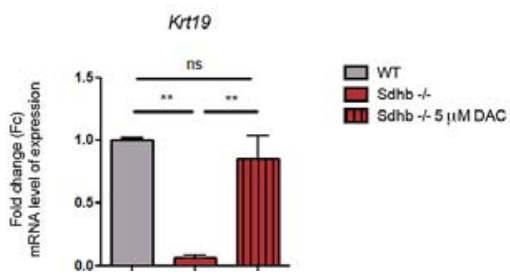

Figure 3: KRT19 loss of expression is driven by hypermethylation. A. Differential methylation rate of $K r t 19$ promoter in $S d h b$ deficient cells compared to WT cells. Fifteen CpG islands were studied in Krt19's promoter. Filled circles figured percentage of methylation of CpG islands. B. KRT19 was studied using immunofluorescence and specific antibody. Labeling was seen is the cytosol together with a non-specific nucleolar staining. Scale bar $20 \mu \mathrm{m}$. C. qRT-PCR analyses showing Krt19 loss of expression in Sdhb-deficient cells compared to WT cells, which is reversed after decitabine treatment. ${ }^{*} p=0.0075$ and $p=0.0025$. Data are shown as fold change relative to WT See also Supplementary Figures 4 and 5.

\section{Krt19 is hypermethylated and downregulated in Sdhb-/- imCC}

In human PPGL, transcriptome and methylome data revealed that the levels of KRT19 mRNA or its promoter's methylation were able to distinguish $S D H B$-mutated tumors from all others tumors (Supplementary Figures 4A and 4B). We thus decided to further investigate KRT19 functions in $S d h b-/-$ imCC. We observed that KRT19 hypermethylation did occur in the murine cell line following $S d h b$ gene inactivation as pointed out by comparing the methylation of $15 \mathrm{CpG}$ islands in $\mathrm{Krt} 19$ promoter by representative reduced bisulfate sequencing (Figure 3A). There was a global 30\% increase in DNA methylation at the Krt19 locus in Sdhb-/imCC compared to WT cells (Supplementary Figure 5). This was associated with the absence of KRT19 protein in $S d h b^{-} /-$imCC (Figure 3B) as a result of a 10-fold decrease in gene expression evaluated by quantitative RT-PCR (Figure 3C). Decitabine treatment led to the re-expression of Krt19 in Sdhb-deficient cells to levels comparable to that observed in WT cells, thus demonstrating that the marked reduction of Krt19 expression was indeed the result of increased methylation (Figure 3D).

\section{KRT19 rescue partially reverses $S d h b-/-$ imCC metastatic hallmarks}

To evaluate the participation of KRT19 to the metastatic phenotype of $S d h b$-deficient cells, we infected cells with a lentivirus encoding the complete $\mathrm{Krt19}$
cDNA. A GFP-expressing lentivirus was used as a control. Although KRT19 lentiviral transduction induced a massive up-regulation of $K r t 19$ mRNA level in both cell types (1,000-fold induction, Supplementary Figure 6A), immunofluorescence analysis showed no differences on protein staining levels detected in WT cells while in $S d h b^{-} /-$imCC, it allowed the detection of the protein at a staining level comparable to that observed in WT cells (Supplementary Figure 6B). We evaluated the impact of KRT19 rescue on the metastatic traits previously described. KRT19 rescue led to a significant (but not complete) inhibition of collective migration with a closure index modestly but significantly decreased from $60 \%$ to $48 \%$ in $S d h b-/-$ imCC and with no effect on WT cells $(26 \%$ in GFP-transduced cells compared to $28 \%$ in KRT19transduced cells) (Figure 4A).

Single cell tracking experiments showed that KRT19 rescue induced a partial but significant reduction of $S d h b^{-/}$- imCC speed (from $16 \mu \mathrm{m} / \mathrm{h}$ to $14 \mu \mathrm{m} / \mathrm{h}$ ), while it had no effect on WT cells $(12 \mu \mathrm{m} / \mathrm{h}$ in both conditions) (Figure 4B). Vector displacement diagrams did not reveal any difference in cells' movement after protein rescue and persistence remained unchanged (Supplementary Figure 7A and 7B). KRT19 rescue had no effect on the invasive capacities of $S d h b-/-$ imCC (Figure 4C-4E) and only mildly delayed their adherence. After $1 \mathrm{~h}, 64 \%$ of cells had adhered as compared to $78 \%$ with GFP transduction (Figures $4 \mathrm{~F}$ and $4 \mathrm{G}$ ) but ultimately, the same percentage of cells adhered at the end of the time-lapse studied (Figure 4G). 

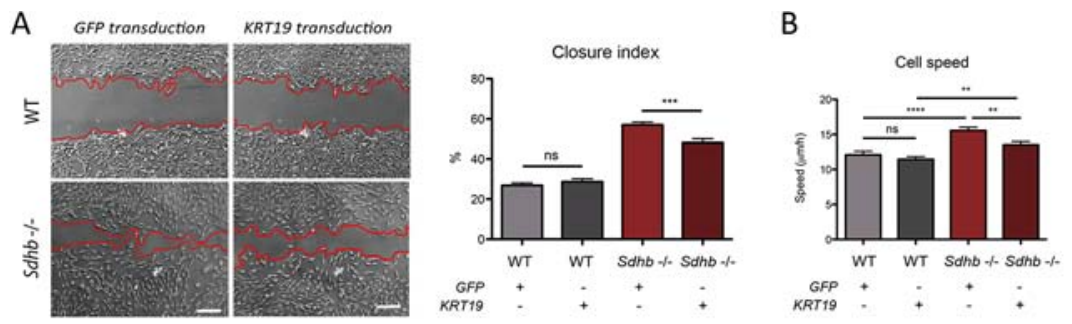

C

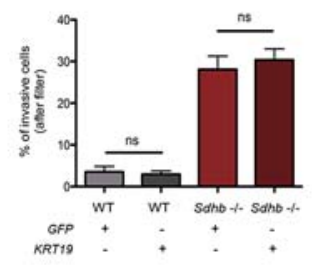

$\mathrm{F}$

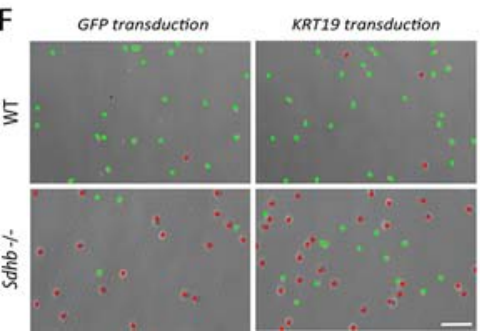

D

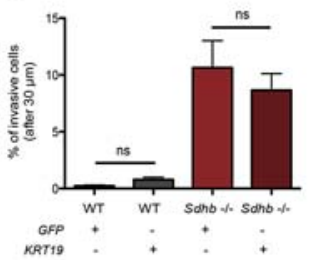

$E$

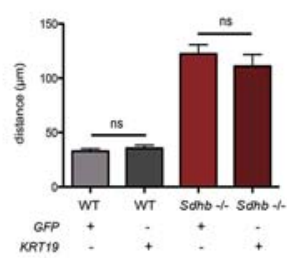

G

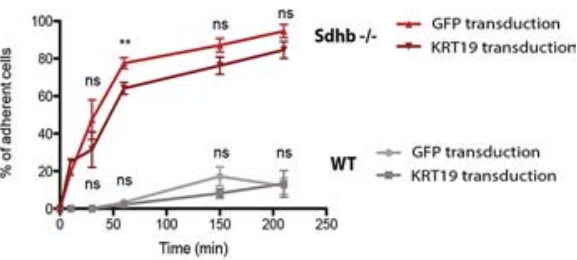

Figure 4: KRT19 rescue after lentiviral transduction and its functional consequences. A. Collective migration was followed using a wound scratch assay. Cells were studied for migration during 10 hours. Migration is represented as the closure percentage of the wound. Scale bar $250 \mu \mathrm{m} .{ }^{* * *} p=0.0002$; ns, non-significant. B. Individual cells speed was estimated by tracking cell nucleus during 12 hours. ${ }^{* *} p=$ 0.0013; ns, non-significant. C. Quantification of cell abilities to pass through the filter. ns, non-significant. D. Quantification of cell invasion, cells were considered invasive beyond $30 \mu \mathrm{m}$. ns, non-significant. E. Mean distance travelled by cells into Matrigel matrix. ns, non-significant. F. Cell adhesion abilities were measured by counting adherent (marked with red dots) versus non-adherent (marked with green dots) cells one hour after plating. Scale bar $125 \mu \mathrm{m}$. G. Adhesion course: quantification of cell adhesion during 210 minutes. ${ }^{* *} p=0.0013$; ns, non-significant See also Supplementary Figures 6 and 7.

\section{KRT19 transient inhibition in WT cells enhances cell's adhesion}

Krt19 expression was inhibited in WT cells using two different siRNAs sequence (Figure 5A). A significant reduction in KRT19 mRNA levels was achieved with only one: si_KRT19_1 (Figure 5B), while both led to a marked decrease in protein levels in a large proportion of transfected cells (Figure 5C), suggesting either transcriptional or translational repression mechanisms.

KRT19 inhibition in WT cells had no effect on global cell migration, as evaluated by the wound scratch assays (Figure 5D). In contrast, it induced a significant increase in cell adherence, one hour after plating (Figure 5E and 5F), which reached 53\% of all cells 4 hours after plating (vs 24\% with scramble siRNAs) (Figure $5 \mathrm{G}$ ).

\section{DISCUSSION}

In the present study, we demonstrate that $S d h b$ inactivation alone is sufficient to promote the in vitro transformation of mouse chromaffin cells into invasive cells. We show that $S d h b$ knockout leads to a molecular reprogramming compatible with the activation of an EMTlike process, as highlighted by both transcriptional and protein modulations of Twist 1/2, Tcf3, Snail, $N$-cadherin and $K r t 19$, previously shown to be differentially expressed in $S D H B$-malignant human PPGL [7]. Our data support the functional role of the epigenetically silenced Krt19 gene, whose repression participates to some of the phenotypic modifications observed in $S d h b$-deficient cells. Altogether, these data establish that $S d h b$ does act as a tumor suppressor gene by promoting epithelial-to-mesenchymal transition and that KRT19 is one actor of this process.

Succinate dehydrogenase (SDH) is composed of four subunits (SDHA-D), each encoded by a different nuclear gene. While mutations in the $4 S D H x$ genes lead to a complete loss of SDH activity and predispose to PPGL, it is still unclear why $S D H B$ inactivation only is responsible for metastatic forms of the disease. Two major hypotheses may explain this difference: i) the coexistence of another genetic or genomic alteration that would act in addition or in synergy with SDHB inactivation; or ii) another, yet unidentified, function of SDHB protein, 

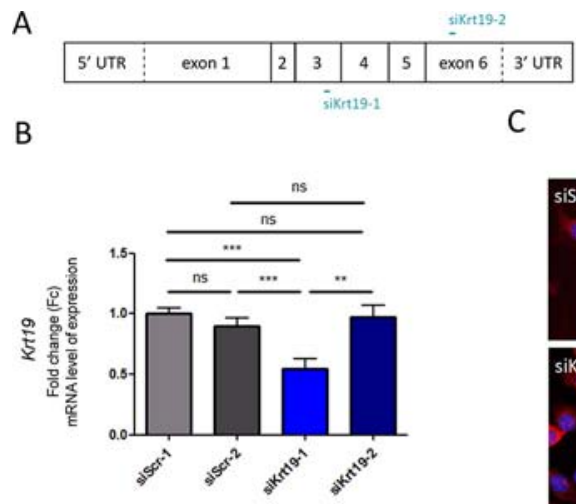

C

D

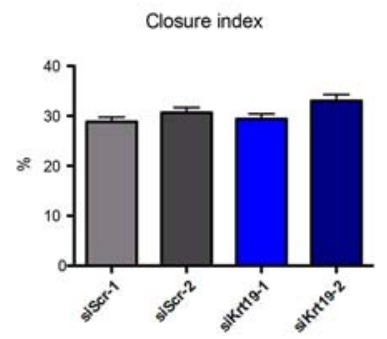

$\mathrm{F}$

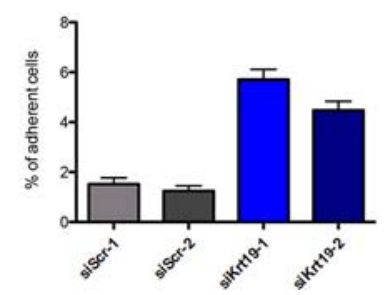

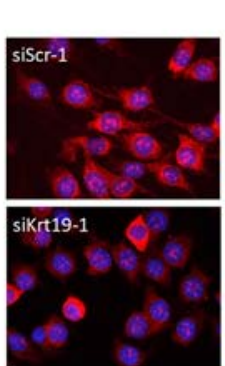

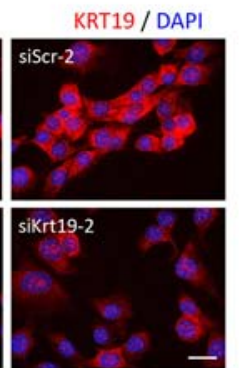

$\mathrm{E}$

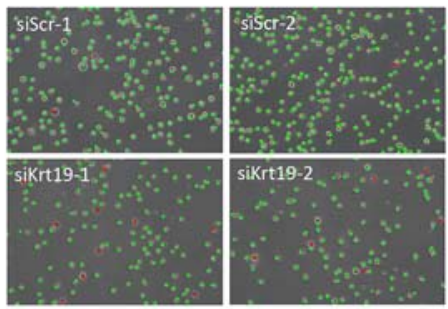

G

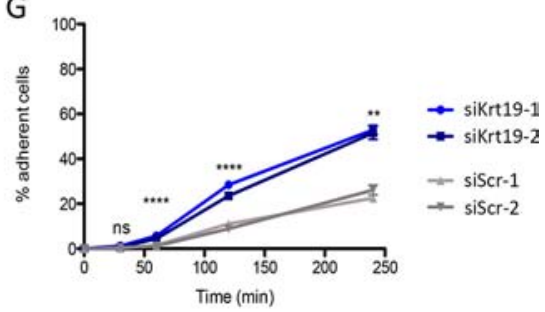

Figure 5: KRT19 transient inhibition by siRNA and its functional consequences. A. Graphical representations of Krt19 mRNA and localization of siRNA interaction. B. KRT19 inhibition was confirmed in WT cells mRNA level using qRT-PCR. As a control cells were independently transfected with the scrambles siRNA. $* * p=0.0030 ; * * *=0.0003$ and $p=0.0007$; ns, non-significant. Data are shown as fold change relative to siScr-1 C. KRT19 inhibition was studied cells at protein level using immunofluorescence. Cells were independently transfected with either one of the scrambles siRNA (siScr-1 or siScr-2) as a control (upper panels), or with one of Krt19 directed siRNA (siKrt19-1 and siKrt19-2) (bottom panels). Scale bar $20 \mu \mathrm{m}$. D. Collective migration was followed using a wound scratch assay. Cells were studied for migration during 10 hours. Migration is represented as the closure percentage of the wound. E. Cell adhesion abilities were measured by counting adherent (marked with red dots) versus non-adherent (marked with green dots) cells one hour after plating. Scale bar $125 \mu \mathrm{m}$. F. Quantification of cell adhesion one hour after plating. G. Adhesion course: quantification of cell adhesion during 240 minutes. ${ }^{* *} p=0.0014$; $* * * * p<0.0001$; ns, non-significant

that would mediate EMT in case of $S D H B$ mutation. Our results in the $S d h b$ knockout cell model are in favor of the second one and suggest that $S D H B$ inactivation is sufficient to induce both PPGL tumorigenesis and the EMT-associated metastatic phenotype.

Referring to 'epithelial-to-mesenchymal transition' in the context of PPGL can be debated, given the non-epithelial state of chromaffin cells. It would be more appropriate to use the term 'neuroendocrineto-mesenchymal transition' (neuroendoMT). In particular, we did not observe the loss of E-cadherin, which is widely used as a hallmark of EMT in epithelial cells, as it is hardly expressed by WT imCC (data not shown). However, we observed the loss of N-Cadherin after Sdhb inactivation. Interestingly, a comparable process has been described in endothelial cells, which are submitted to an EMT-like phenomenon, occurring during fibrosis. This process, referred to as 'endothelial-to-mesenchymal transition' (endoMT) is associated with the loss of the only expressed cadherin: the VE-cadherin $[8,27]$.

In human tumors and in $S d h b^{-} /-$imCC, SDHB loss is responsible for succinate dehydrogenase inactivation, succinate accumulation, and subsequent inhibition of 2-oxoglutarate dependent dioxygenases [22, 24]. Of the dioxygenases described to date, TETs enzymes are responsible for DNA demethylation, and are inhibited by succinate, leading to global hypermethylation of more than 4000 genes, including KRT19. Intermediate filaments have been described as being implicated in cell migration and adhesion $[11,18]$ as well as in cell invasion $[14,18]$. 
By being one of the most significantly hypermethylated, and one of the most significantly down-regulated gene, KRT19 was a pertinent candidate to decipher the still misunderstood link between $S D H B$ deficiency, hypermethylation, EMT and malignancy. This work identifies KRT19 as one of the important actors implicated in migratory and adhesive properties of $S d h b-/-$ imCC and thus in $S D H B$ associated metastatic phenotype. However, in vitro results after KRT19 rescue in $S d h b$-deficient cells show that KRT19 repression has only a very mild impact on invasion (despite a trend to a reduced ability to pass through the filter and of the percentage of invasive cells). Altogether, our data show that in addition to KRT19, other EMT actors are implicated in the establishment of the metastatic skills we observed. Further studies will be needed to investigate other target candidates and search for eventual synergic effects with KRT19.

With the validation of the first experimental cellular model that mimics the $S D H B$-related metastatic phenotype in chromaffin cells, this study opens a new research area for $S D H B$-related oncongenesis. We show that SDHB loss alone induces a neuroendoMT phenomenon associated with increased metastatic abilities and likely explaining the bad prognosis caused by $S D H B$ mutations in patients. KRT19 is one of the partners implicated in the establishment of the mesenchymal phenotype and in the process that drives PPGL malignancy.

\section{MATERIALS AND METHODS}

\section{Cell line}

Wild-type ( $\left.S d h b^{\text {lox/lox}}\right)$ immortalized mouse chromaffin cells (imCC) were isolated from the adrenal medulla of a genetically modified mouse harboring LoxP sites on both sides of exon 2 of the Sdhb gene [28]. After spontaneous in vitro immortalization, $S d h b$ knockout was performed using an adenovirus expressing Cre recombinase [24]. Cell morphology was assessed by lucent phase microscopy (Supplementary Figure 1A) and by immunostaining of their tubulin cytoskeleton (Supplementary Figure 1B).

\section{Cell culture}

imCC were cultured in DMEM (Dulbecco Modified Eagle Medium, Gibco) with 4.5\% FBS (Fetal bovine serum, Gibco) and 1\% antibiotics (penicillin/streptomycin, Gibco). Cells were grown at $37^{\circ} \mathrm{C}$, in $5 \% \mathrm{CO}_{2}$. Experiments were performed using one of the two imCC clones: clone 8 and its WT counterpart [24]. Except when mentioned, all experiments were performed using this regular medium, on regular plastic plates and dishes, without coating.

\section{Single cell tracking}

Exponentially growing cells were seeded at a density of $5 \times 10^{4}$ in a six-well plate. After $24 \mathrm{~h}$ of incubation, the cells were imaged every 4 min for $12 \mathrm{~h}$. The nucleus of each cell was manually tracked with the Manual Tracking plugin for ImageJ. Experiments were performed three times, with at least 25 cells tracked each time.

\section{Invasion assay}

A Matrigel matrix was previously polymerized in a Transwell system (BD Biosciences); $5 \times 10^{4}$ cells were seeded on in complete medium and left to adhere for $4 \mathrm{~h}$. After 3 washes in medium without FBS, cells were allowed to invade the matrix during 5 days, at $37^{\circ} \mathrm{C}$, with $5 \% \mathrm{CO}_{2}$. Medium with FBS was added on top of the Matrigel matrix as a stimulus. After $1 \mathrm{~h}$ of calcein staining, living cells were followed using confocal microscopy. For each experiment, each sample was performed in duplicate and 3 areas of the Transwell were studied. Experiments were performed three times.

\section{Adhesion assay}

After complete detachment from their plate using trypsin-EDTA $0.05 \%$ (Gibco), $5 \times 10^{4}$ cells were plated in 6-wells dishes. Matrige $\mathrm{l}^{\circledR}$ coating was performed with pure Matrigel $^{\circledR}$ (BD Biosciences), allowed to polymerized 30 minutes at $37^{\circ} \mathrm{C}$. Laminin coating was performed using $100 \mu \mathrm{g} / \mathrm{ml}$ solution, allowed to polymerized 1 hour at room temperature. Cell adhesion was followed by taking picture during $3 \mathrm{~h}$. Adherent and non-adherent cells were identified on the basis of their morphology (round and refractive cells were considered as non-adherent). Cells were marked on the image with a green dot if considered as non-adherent and with a red dot if considered as adherent and the respective numbers of dots were quantified. Experiments were performed three times.

\section{Human tumors methylome analysis}

KRT19 methylation data was a part of global DNA methylation study performed on a 145 samples PCC/PGL collection the Illumina Infinium HumanMethylation27 assay, as described elsewhere [24].

\section{Human tumors transcriptome analysis}

KRT19 level of expression data were a part of EMT study performed on 188 samples of PCC and PGL and using the HG-U133 Plus 2.0 Affymetrix Gene Chip arrays, as described elsewhere [7].

\section{imCC transcriptome analysis}

Gene expression profiles of wild-type and $S d h b^{-/-}$ imCC c8 were assessed in duplicates using the Gene Chip ${ }^{\circledR}$ Mouse Gene 1.0 ST Array. Data are available as Array Express entry E-MTAB-3403. 


\section{imCC methylome analysis}

Krt19 methylation data was a part of global DNA methylation study performed on imCC using Reduced Representation Bisulphite Sequencing technique (RRBS), as previously described [24].

\section{Decitabine treatment}

A daily fresh prepared and filtered solution of decitabine (A3656, Sigma Aldrich) was used to treat cells, during 5 days. Drug $(0.25 \mathrm{mg}$ ) was resuspended in DMSO and diluted in culture medium. A classical $(5 \mu \mathrm{M})$ [29] dose was used; not-treated cells received medium with equivalent amount of DMSO only.

\section{Quantitative Real-Time (qRT)-PCR}

Total RNAs were extracted from cell pellets using RNA Extract II kit $^{\circledR}$ (Macherey-Nagel), as described by the manufacturer. Three $\mu \mathrm{g}$ of RNA were treated with DNase Amp Grade and EDTA (Invitrogen) during $10 \mathrm{~min}$ at $65^{\circ} \mathrm{C}$. Reverse transcription was performed on $1.5 \mu \mathrm{g}$ of RNA, using random primers and Superscript Reverse transcriptase (Invitrogen), during $1 \mathrm{~h}$ at $42^{\circ} \mathrm{C}$. RNA concentrations were determined with a NanoVue Plus (GE Healthcare ${ }^{\circledR}$ ) and subjected to qRT-PCR in triplicate, using PTC-200 DNA Engine ${ }^{\circledR}$ Thermal Cycler. Normalization was performed with Ubc and $18 \mathrm{~S}$ amplifications, and comparisons were calculated using the $\Delta \Delta \mathrm{Ct}$ method. Primers' sequences were as follows:

Ubc: F 5'-AGCCCAGTGTTACCACCAAG-3'; R 5'-ACCCAAGAACAAGCACAAG-3';

18S: F 5'-CGCGGTTCTATTTTGTTGGT-3'; R 5'-AACCATAAACGATGCCGAC-3';

Twist1: F 5'-CTGGACTCCAAGATGGCAAGC TG-3'; R 5'-ACCTAGATGTCATTGTTTCCAGAGAA-3'

Twist2: F 5'-CATGTCCGCCTCCCACTA-3'; R

5'-ACCTTGTGGCTCCTCATGAC-3'

Tcf3: F 5'-TGGCACTTACAGTGGGACTTC-3'; R

5'-GTTGAGGGGCTAGGTGAGAA-3'

Snail: F 5'-TGGAAAGGCCTTCTCTAGGC-3'; R

5'-CCACTGCAACCGTGCTTTT-3'

Krt19: F 5'-GGTGCCACCATTGACAACTC-3'; R

5'-GAGACAGAACACGCCTTGC-3';

Cdh2: F5'-GAAGATGTTTACAGCGCAGTCTT-3'; R 5'-AAGTTCAGTATGAAAGCAGCGAG-3'

\section{Immunofluorescence assay}

For immunofluorescence assays, cells plated on glass slides were washed in PBS and fixed for $10 \mathrm{~min}$ in ice-cold paraformaldehyde $4 \%$ or methanol $100 \%$. Antigen retrieval was performed with Tris-EDTA buffer (1 mM EDTA $\mathrm{pH}=8,0.005 \%$ Twin $20 ; \mathrm{pH}=9$ ) or with citrate buffer $(0.01 \mathrm{M}$ sodium citrate, $0.05 \mathrm{M}$ citric acid, $\mathrm{pH}=6)$, during
45 seconds in a microwave. After blockade of aspecific sites ( $1 \%$ BSA, $0.1 \%$ Triton), primary antibodies were incubated for $90 \mathrm{~min}$ at room temperature. The antibodies used were: anti-SDHA (Abcam, Ab14715), anti-SNAI1 (Abcam, Ab85931), anti-KRT19 (Thermo RB9021), anti-N-cadherin (Abcam, Ab12221), and anti $\alpha$-tubulin (Sigma-Aldrich). Secondary antibodies, conjugated with Alexa Fluor 488 or with Alexa Fluor 594 (1/2000, Invitrogen), were incubated for $1 \mathrm{~h}$, at room temperature in the dark. Slides were finally mounted in Vectashield, containing DAPI, for a blue nuclear staining. Acquisitions were performed using Axioimager Z1 Zeiss, with apotome system.

\section{Western blot analysis}

Total proteins were extracted, in Tris-EDTA buffer (Tris $\mathrm{NaCl} \mathrm{pH}=7.4,0.5 \% \mathrm{NP}-40,0.5 \%$ deoxycholic acid and $5 \mathrm{mM}$ EDTA) containing proteases inhibitors cocktail. Protein concentrations were determined with Bradford colorimetric method. $50 \mu \mathrm{g}$ were resolved on NuPAGE ${ }^{\circledR}$ Novex 4-16\% Bis-Tris gradient gel (Invitrogen), transferred on PVDF membrane. After blocking in PBSmilk solution (5\%), membranes were incubated with specific primary antibodies, followed by incubation with corresponding HRP-conjugated secondary antibodies. Revelation was performed using ECL Plus (Pierce). The antibodies used were: anti-SDHA (Abcam, Ab14715), anti-SDHB (Sigma, HPA002868), anti-SNAI1 (Abcam, Ab85931), and anti-TCF3 (Abcam, Ab69999).

\section{Promoter methylation analysis}

Genomic DNA was treated with bisulfite, using Epitect Bisulfite kit (Qiagen), following manufacturer's recommendations. Fifteen $\mathrm{CpG}$ island, of Krt19's promoter, were studied by pyrosequencing using Pyromark Q24 instrument and software, and following manufacturer's recommendations.

\section{Plasmid construction}

GFP (Green Fluorescent Protein) and KRT19 (Keratin19) cDNA were inserted in a lentiviral shuttle plasmid by Gateway recombination (Life Technologies, Saint-Aubin, France) under the transcriptional control of the PGK (Phosphoglycerate Kinase) promoter. The recombinant lentiviral genome contained a selfinactivating deletion of the left LTR and the central polypurine tract/central termination sequence (cPPT/CTS) and was previously described [30].

\section{Virus production and cell transduction}

Recombinant lentiviral particles were produced by transient transfection of HEK-293T cells as previously described [30]. Viral supernatants were concentrated by 
ultracentrifugation at $70,000 \mathrm{~g}$ for $90 \mathrm{~min}$ at $4{ }^{\circ} \mathrm{C}$. Finally, to achieve a 1,000-fold concentration of the initial supernatant, viral pellets were resuspended in a minimal volume of PBS. Aliquots of $5-10 \mu \mathrm{l}$ were then stored at $-80^{\circ} \mathrm{C}$ until further use. Total particle concentration of the viral stocks was estimated by quantification of the p24 capsid protein using RETRO-TEK HIV-1 p24 Antigen ELISA kit (ZeptoMetrix, Buffalo, New York, United States) according to the manufacturer's instructions. For transduction of $5 \times 10^{4}$ cells, a multiplicity of transduction (MOI) of $2 \mathrm{ng}$ of p24 protein/cell was used.

\section{Wound scratch assay}

Cells were seeded at confluence in 6-well dishes. Twenty-four hours after plating, a wound was made using a $10 \mu \mathrm{L}$ tip and cells were allowed to migrate for $10 \mathrm{~h}$. Relative cell migration was established by calculating the closure index, based on the analysis of at least 25 pictures for each time point and condition, using ImageJ software, as described [24]. The experiments were performed in triplicates.

\section{siRNA transient transfection}

For KRT19 inhibition in WT cells, custom siRNA were designed and tested. Two of them were chosen as they induced an important Krt19 inhibition, siKrt19-1: 5' UGGCCUACCUGAAGAAGAA 3' and siKrt19-2: 5' GCCAGAACCAGGAGUAUAA 3'. siRNA were then transfected using HiPerfect transfection reagent (Qiagen), along with cell plating, and cells were collected 36 hours after transfection. Two different negative controls siRNA were used, as they did not react with any known mouse mRNA. Scrambles siRNA were design as follow, siScr-1: 5' UACGCAUUCGUCAUUGCUA 3' and siScr-2: 5' UCGAAGUAUUCCGCGUACG 3'.

\section{Statistical analysis}

All statistical analyses were performed using Graph Pad Prism software. Data are represented as mean (of at least 3 independent experiments) \pm SEM (standard error of the mean).

\section{ACKNOWLEDGMENTS AND FUNDING}

We would like to thank "La Fondation ARC" (DOC20130606831), and "Le Cancéropôle Ile-de-France" (2013-2-AD-02-UP 5-1), which allowed this project to be performed thanks to its support.

We are also grateful to by La Ligue Nationale Contre le Cancer, Cancéropôle Ile-de-France, INCa and Pair Mélanome, which supported MD and LL.

\section{CONFLICTS OF INTEREST}

The authors have declared that no conflict of interest exists.

\section{GRANT SUPPORT}

This work was supported by the Programme Hospitalier de Recherche Clinique grant COMETE 3 (AOM 06 179) and the Agence Nationale de la Recherche (ANR2011- JCJC-00701 MODEOMAPP). This project was also funded by the European Union Seventh Framework Programme (FP7/2007-2013) under grant agreement number 259735 and by the Plan Cancer: Appel à projets Epigénétique et Cancer 2013 (EPIG201303 METABEPIC).

\section{REFERENCES}

1. Dahia PL. Pheochromocytoma and paraganglioma pathogenesis: learning from genetic heterogeneity. Nat Rev Cancer. 2014; 14:108-119.

2. Cascon A, Comino-Mendez I, Curras-Freixes M, de Cubas AA, Contreras L, Richter S, Peitzsch M, Mancikova $\mathrm{V}$, Inglada-Perez L, Perez-Barrios A, Calatayud M, Azriel S, Villar-Vicente R, Aller J, Setien F, Moran S, et al. Whole-exome sequencing identifies MDH2 as a new familial paraganglioma gene. Journal of the National Cancer Institute. 2015; 107.

3. Favier J, Amar L, Gimenez-Roqueplo AP. Paraganglioma and phaeochromocytoma: from genetics to personalized medicine. Nature reviews Endocrinology. 2015; 11:101-11.

4. Amar L, Baudin E, Burnichon N, Peyrard S, Silvera S, Bertherat J, Bertagna X, Schlumberger M, Jeunemaitre X, Gimenez-Roqueplo AP, Plouin PF. Succinate dehydrogenase $\mathrm{B}$ gene mutations predict survival in patients with malignant pheochromocytomas or paragangliomas. The Journal of clinical endocrinology and metabolism. 2007; 92:3822-3828.

5. Castro-Vega LJ, Letouze E, Burnichon N, Buffet A, Disderot PH, Khalifa E, Loriot C, Elarouci N, Morin A, Menara M, Lepoutre-Lussey C, Badoual C, Sibony M, Dousset B, Libe R, Zinzindohoue F, et al. Multi-omics analysis defines core genomic alterations in pheochromocytomas and paragangliomas. Nature communications. 2015; 6:6044.

6. Castro-Vega LJ, Lepoutre-Lussey C, Gimenez-Roqueplo AP, Favier J. Rethinking pheochromocytomas and paragangliomas from a genomic perspective. Oncogene. 2015. doi: 10.1038/ onc.2015.172. [Epub ahead of print]

7. Loriot C, Burnichon N, Gadessaud N, Vescovo L, Amar L, Libe R, Bertherat J, Plouin PF, Jeunemaitre X, GimenezRoqueplo AP, Favier J. Epithelial to mesenchymal transition is activated in metastatic pheochromocytomas and paragangliomas caused by SDHB gene mutations. 
The Journal of clinical endocrinology and metabolism. 2012; 97:E954-962.

8. Iwano M, Plieth D, Danoff TM, Xue C, Okada H, Neilson EG. Evidence that fibroblasts derive from epithelium during tissue fibrosis. The Journal of clinical investigation. 2002; 110:341-350.

9. Thiery JP. Epithelial-mesenchymal transitions in tumour progression. Nat Rev Cancer. 2002; 2:442-454.

10. Kalluri R, Weinberg RA. The basics of epithelialmesenchymal transition. The Journal of clinical investigation. 2009; 119:1420-1428.

11. Seltmann K, Roth W, Kroger C, Loschke F, Lederer M, Huttelmaier S, Magin TM. Keratins mediate localization of hemidesmosomes and repress cell motility. The Journal of investigative dermatology. 2013; 133:181-190.

12. Schweizer J, Bowden PE, Coulombe PA, Langbein L, Lane EB, Magin TM, Maltais L, Omary MB, Parry DA, Rogers MA, Wright MW. New consensus nomenclature for mammalian keratins. The Journal of cell biology. 2006; 174:169-174.

13. Androulakis N, Agelaki S, Perraki M, Apostolaki S, Bozionelou V, Pallis A, Kalbakis K, Xyrafas A, Mavroudis D, Georgoulias V. Clinical relevance of circulating CK-19mRNA-positive tumour cells before front-line treatment in patients with metastatic breast cancer. British journal of cancer. 2012; 106:1917-1925.

14. Crowe DL, Milo GE, Shuler CF. Keratin 19 downregulation by oral squamous cell carcinoma lines increases invasive potential. Journal of dental research. 1999; 78:1256-1263.

15. Caren H, Djos A, Nethander M, Sjoberg RM, Kogner P, Enstrom C, Nilsson S, Martinsson T. Identification of epigenetically regulated genes that predict patient outcome in neuroblastoma. BMC cancer. 2011; 11:66.

16. Nozato $M$, Kaneko S, Nakagawara A, Komuro $H$. Epithelial-mesenchymal transition-related gene expression as a new prognostic marker for neuroblastoma. International journal of oncology. 2013; 42:134-140.

17. Morris MR, Gentle D, Abdulrahman M, Clarke N, Brown M, Kishida T, Yao M, Teh BT, Latif F, Maher ER. Functional epigenomics approach to identify methylated candidate tumour suppressor genes in renal cell carcinoma. British journal of cancer. 2008; 98:496-501.

18. Ju JH, Yang W, Lee KM, Oh S, Nam K, Shim S, Shin SY, Gye MC, Chu IS, Shin I. Regulation of cell proliferation and migration by keratin-induced nuclear import of early growth response- 1 in breast cancer cells. Clin Cancer Res. $2013 ; 19: 4335-4346$.

19. Tripathi MK, Misra S, Chaudhuri G. Negative regulation of the expressions of cytokeratins 8 and 19 by SLUG repressor protein in human breast cells. Biochemical and biophysical research communications. 2005; 329:508-515.

20. Navarro A, Yin P, Monsivais D, Lin SM, Du P, Wei JJ, Bulun SE. Genome-wide DNA methylation indicates silencing of tumor suppressor genes in uterine leiomyoma. PloS one. 2012; 7:e33284.
21. Paiva F, Duarte-Pereira S, Costa VL, Ramalho-Carvalho J, Patricio P, Ribeiro FR, Lobo F, Oliveira J, Jeronimo C, Henrique R. Functional and epigenetic characterization of the KRT19 gene in renal cell neoplasms. DNA and cell biology. 2011; 30:85-90.

22. Xiao M, Yang H, Xu W, Ma S, Lin H, Zhu H, Liu L, Liu Y, Yang C, Xu Y, Zhao S, Ye D, Xiong Y, Guan KL. Inhibition of alpha-KG-dependent histone and DNA demethylases by fumarate and succinate that are accumulated in mutations of $\mathrm{FH}$ and $\mathrm{SDH}$ tumor suppressors. Genes \& development. 2012; 26:1326-1338.

23. Killian JK, Kim SY, Miettinen M, Smith C, Merino M, Tsokos M, Quezado M, Smith WI Jr., Jahromi MS, Xekouki P, Szarek E, Walker RL, Lasota J, Raffeld M, Klotzle B, Wang Z, et al. Succinate dehydrogenase mutation underlies global epigenomic divergence in gastrointestinal stromal tumor. Cancer discovery. 2013; 3:648-657.

24. Letouze E, Martinelli C, Loriot C, Burnichon N, Abermil N, Ottolenghi C, Janin M, Menara M, Nguyen AT, Benit P, Buffet A, Marcaillou C, Bertherat J, Amar L, Rustin P, De Reynies A, et al. SDH mutations establish a hypermethylator phenotype in paraganglioma. Cancer cell. 2013; 23:739-752.

25. Morin A, Letouze E, Gimenez-Roqueplo AP, Favier J. Oncometabolites-driven tumorigenesis: From genetics to targeted therapy. International journal of cancer. 2014; 135:2237-48.

26. Reymond N, d'Agua BB, Ridley AJ. Crossing the endothelial barrier during metastasis. Nature reviews. 2013; 13:858-870.

27. Nakajima $Y$, Yamagishi $T$, Hokari S, Nakamura $H$. Mechanisms involved in valvuloseptal endocardial cushion formation in early cardiogenesis: roles of transforming growth factor (TGF)-beta and bone morphogenetic protein (BMP). The Anatomical record. 2000; 258:119-127.

28. Lepoutre-Lussey C, Thibault C, Buffet A, Morin A, Badoual C, Benit P, Rustin P, Ottolenghi C, Janin M, Castro-Vega LJ, Trapman J, Gimenez-Roqueplo AP, Favier J. From Nf1 to Sdhb knockout: Successes and failures in the quest for animal models of pheochromocytoma. Mol Cell Endocrinol. 2015. doi: 10.1016/j.mce.2015.06.027. [Epub ahead of print]

29. Tsai HC, Li H, Van Neste L, Cai Y, Robert C, Rassool FV, Shin JJ, Harbom KM, Beaty R, Pappou E, Harris J, Yen RW, Ahuja N, Brock MV, Stearns V, Feller-Kopman D, et al. Transient low doses of DNAdemethylating agents exert durable antitumor effects on hematological and epithelial tumor cells. Cancer cell. 2012; 21:430-446.

30. Bemelmans AP, Bonnel S, Houhou L, Dufour N, Nandrot E, Helmlinger D, Sarkis C, Abitbol M, Mallet J. Retinal cell type expression specificity of HIV-1-derived gene transfer vectors upon subretinal injection in the adult rat: influence of pseudotyping and promoter. The journal of gene medicine. 2005; 7:1367-1374. 